\title{
Taming Twisted Cubes
}

\begin{tabular}{|c|c|c|c|c|}
\hline \multirow{2}{*}{ Baumann } & Eric & lonn Maco & & \\
\hline & СПL ПIISCTUII & Jual IVIaso & FIEx Dumara & viad iviericaniu \\
\hline $\begin{array}{l}\text { Jacobs University } \\
\text { Bremen, Germany }\end{array}$ & $\begin{array}{l}\text { KEYW Corp. } \\
\text { Hanover, MD, US }\end{array}$ & $\begin{array}{c}\text { CREAF } \\
\text { Barcelona, Spain }\end{array}$ & $\begin{array}{l}\text { rasdaman GmbH } \\
\text { Bremen, Germany }\end{array}$ & $\begin{array}{l}\text { rasdaman GmbH } \\
\text { Bremen, Germany }\end{array}$ \\
\hline & & & & \\
\hline -university.de & rp.com & & com & an.com \\
\hline
\end{tabular}

\begin{abstract}
Spatio-temporal grid data form a core structure in Earth and Space sciences alike. While Array Databases have set out to support this information category they only offer integer indexing, corresponding to equidistant grids. However, often grids in reality have irregular structures, such as raw satellite swath data.

We present an approach to modeling spatio-temporal regular and non-regular grids in a coherent manner, suitable for querying, transporting, and storing such data while remaining format independent. We briefly describe an implementation based on the combination of a relational and an array DBMS. Our model is currently under adoption as an international standard by OGC and ISO.
\end{abstract}

\section{Categories and Subject Descriptors}

[Information Systems]: Information Systems Applications Spatial-temporal systems.

\section{General Terms}

Spatio-temporal grid, raster, array, coverage, OGC, Big Data

\section{INTRODUCTION}

Spatio-temporal grid data form a core structure in Earth and Space sciences alike, appearing as satellite imagery, meteorological simulations, telescope data, etc. in huge and ever-growing amounts. Such grids can be regular (like ortho imagery) or highly irregular (such as raw satellite swath data) - a variability which creates a substantial diversity in data structures and, consequently, complexity in handling them. Examples of irregular grids include raw satellite and aerial data (Fig. 1) and simulation data (Fig. 2); in fact, there is ample reference in literature, e.g., [27][32][31][12]. Situations get worse once more than just the usual two horizontal dimensions are involved.

We illustrate this by way of two common situations. The first addresses timeseries over raw swath data, i.e., non-rectified Level0 satellite imagery. In the most general case, Lat/Long coordinates require an enumeration of pixel positions through an associated 2D array of actual coordinates (Fig. 1). A datacube definition capable of holding such data will have the following axes: Lat/Long, which both are irregular and "distorted"; and Time, which is irreg-

\footnotetext{
Permission to make digital or hard copies of all or part of this work for personal or classroom use is granted without fee provided that copies are not made or distributed for profit or commercial advantage and that copies bear this notice and the full citation on the first page. Copyrights IRUMLGSDUVIFRP SRQHQWRILUVWZ RLNIP XWMHIKRQRUHG

Copyright is held by the owner/author(s).

GeoRich'16, June 26-July 01 2016, San Francisco, CA, USA

2016 ACM. ISBN 978-1-4503-4309-1/16/06. . \$15.00.

http://dx.doi.org/10.1145/2948649.2948650

(c) (i) (2)

This work is licensed under a Creative Commons Attribution-ShareAlike

International 4.0 License.
}

ular as well due to the varying overpass and acquisition times.

Our second example is a 4-D spatio-temporal grid as generated through weather forecast simulations. Such a "weather datacube" has the following axes: Lat/Long, which are regular; Time, which is regular due to the fixed simulation timesteps; and Height, which is irregular as well as simulation resolution decreases with distance from ground.

Data interchange and service functionality suffer substantially from the lack of a uniform model which formalizes the variety of grids occurring in a practical, easy-to-use way and also allows for efficient implementation of data services. While Array Databases have set out to support this information category - see, e.g., [9][25] - they only offer integer indexing, corresponding to equidistant grids. Typically, therefore, specialized data formats such as NetCDF [24] and HDF [18] are being used to represent such data, however without a corresponding service interface. The OGC Web Coverage Service (WCS) [6] offers such an interface, but its underlying grid coverage model [8] is not completely precise, and some non-regular grid types can be described only inefficiently.

Aside from a concise, comprehensive, and efficient modeling, another important requirement is a description concise enough to derive automated conformance tests [13]. Conversely, it must be abstract enough to not prescribe any particular data format.
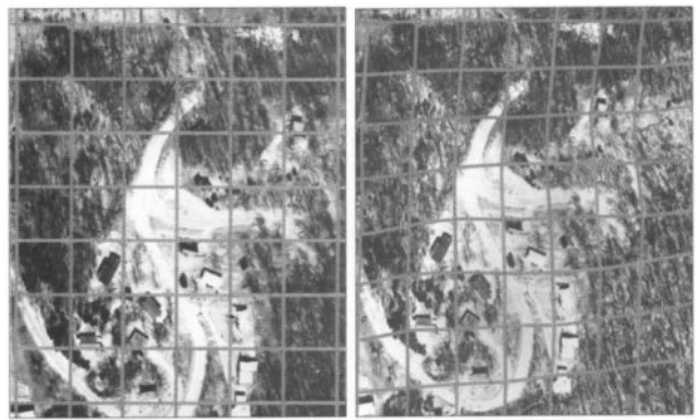

Fig. 1. Original and orthorectified aerial image [19]

In this contribution, we present a model closing this gap: it can represent regular and non-regular grids in a coherent manner, suitable for querying, transporting, and storing such data while not anticipating any specific encoding. Key components have been implemented in the rasdaman Array DBMS, comprehensive support is under way. The model, which is based on earlier findings [20], is currently under adoption as an international standard by OGC and ISO as extension to the widely implemented OGC coverage standard [8]. Service interfaces like the OGC Web Coverage Service (WCS) [6] and Web Coverage Processing Service (WCPS) [7] standards rely on the coverages.

The remainder of this contribution is organized as follows. In the next section, we discuss the state of the art. In Section 3 we intro- 
duce our proposal, the Coverage Implementation Schema (CIS). In Section 4 we describe its implementation and how such data can be served in a standards context. Section 5 concludes the plot.

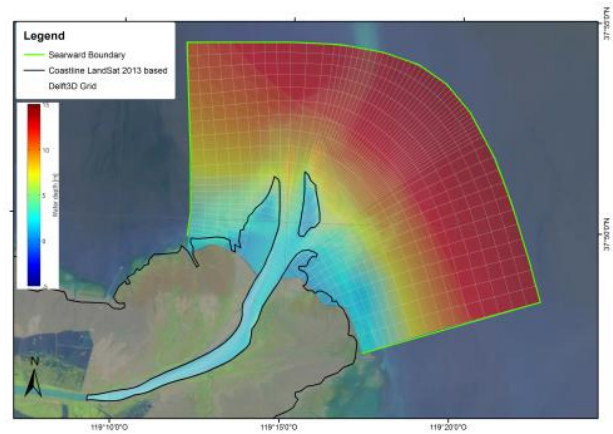

Fig. 2. Curvilinear grid for 3-D bathymetric modeling [16]

\section{STATE OF THE ART}

Gridfields is an algebraic processing approach for regular and irregular grids [21]. It is constructed in a way that makes it largely agnostic of the grid underlying a data set. As such, it does not offer a conceptualization of different grid types. Therefore it is complementary to our approach, and well combinable.

The OGC WMS standard [15] has found wide adoption as a map frontend suitable for zoom and pan on regularly gridded map overlays. Curvilinear grid support is added to WMS by ncWMS [10]. However, this is tied to the representation of the CF-NetCDF file format [24] and lacks an underlying conceptual model.

The coverage paradigm has been established to unify treatment of regular and non-regular grids, point clouds, and general meshes. Its abstract definition is given by ISO 19123 [1], a concrete, interoperable implementation model is given by the OGC Coverage Implementation Schema (CIS) standard [8] whose XML encoding relies on GML (Geography Markup Language), a standardized XML language for geographic objects [28] while allowing encodings in any other suitable format, such as JSON, RDF, GeoTIFF, NetCDF, etc. According to CIS, a coverage is defined by essentially four constituents:

- The domain set describing all locations, called direct positions, for which the coverage contains values.

- The range set listing the value of each direct position.

- The range type defining the type common to all range values.

- A metadata container which is not defined further in the coverage model; this allows transporting any application specific additional information.

From GML coverages also inherit the distinction between (regular) Rectified Grids and (irregular) Referenceable Grids. Aside from the non-intuitive naming this subdivision is rather coarse as we will discuss below.

While the original GML 3.2.1 grid is concise only on Rectified Grids (Fig. 3 top right and left), its extension GML 3.3 adds concrete definitions for several Referenceable Grid subtypes [29]: ReferenceableGridByVector allows all grid axes to have a varying spacing (Fig. 3 bottom left), using a data structure that keeps the individual stepping along each axis as an additional vector for each axis; ReferenceableGridByArray retains a grid topology, but with each grid coordinate sitting at individual locations (Fig. 3 right). Note that each such generalization steps requires more coordinate information to be stored explicitly; in the "ByArray" case this effectively means storing a complete coordinate for each pixel which effectively can lead to more than double the disk space required. A further grid type, ReferenceableGridByTransformation, defines an algorithmic mapping from locations to array coordinates.
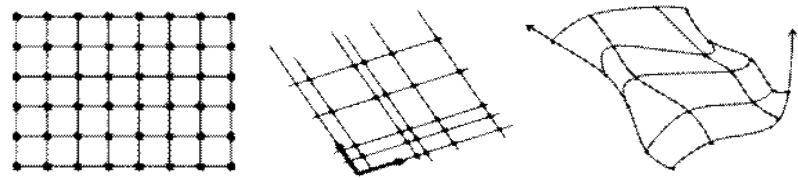

Fig. 3. Some grid types: equidistant (left), irregular (center), distorted (right) [28]

While the combination of CIS and GML 3.x [28][29] allows to represent the classical categorization into regular and irregular grids it misses mixed approaches, for example regular spatial Lat/Long axes combined with an irregular time axis (Fig. 4 left) or some warped space combined with an independently irregular time axis (Fig. 4 right). Note that the distortion direction upwards is in a spatial direction, whereas the vertical axis denotes time and so is in a different direction - an effect due to the limitations of drawing 4-D spaces in 2-D.
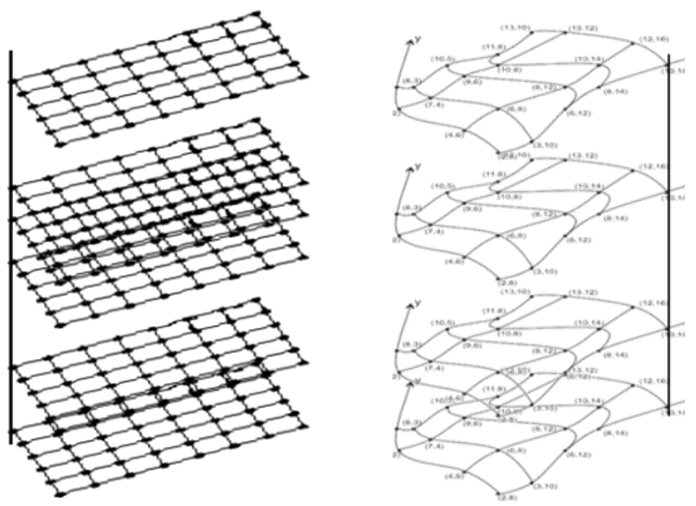

Fig. 4. Sample grids combining regular and irregular axes (left) and irregular axes and "distorted" grids (right); time axis is vertical [20]

Our model overcomes this by not characterizing the grid in total, but each axis individually; this allows combining regular with irregular axes, for example. As we will show, this leads to a both concise and efficient representation.

In terms of grid data processing, groundbreaking work has been done by Tomlin at al [33] in establishing a 2-D map algebra, extended to 3-D by Mennis [22]. Array Algebra has been established as a foundation for array query languages [4]. All these have in common that they only consider simple regular grids, but no irregularity whatsoever.

Hence, to the best of our knowledge this is the first time that nonregular grids are addressed in specifically.

\section{AXIS-CENTRIC GRID MODELING}

In this section, we present our approach to an adaptive modeling of grids with a classification schema that is not based on the grid overall, but rather on a combination of axes with individual 
properties. An overall grid, as usual, is given by an ordered sequence of axes, identified by a locally unique name.

In a coverage, the domain set contains an n-D Coordinate Reference System (CRS) reference which defines the axes used. We adopt the notation of the OGC CRS Name Type Specification [14] which, in addition to referencing predefined CRSs like those of the EPSG collection, provides for a composition mechanism. For example, the following CRS URL defines the concatenation of a spatial Lat/Long/height CRS with time:

\section{http://www.opengis.net/def/crs-compound? 1=http://www.opengis.net/def/crs/EPSG/0/4979 \& \\ 2=http://www.opengis.net/def/crs/OGC/0/AnsiDate}

The numbered keys define the axis/CRS sequence. Further, such a CRS defines axis labels, which a coverage for convenience and efficient evaluation provides locally; in our case, these are Lat, $L o n g, h$, and date. The number of axes defines the topological dimension of a coverage ${ }^{1}$. Additionally, as we consider only finite grids, we require a lower and upper bound along each grid axis.

We introduce each axis type informally in turn, walking from the special axis types to increasing generality. In the final synopsis we combine and formalize them.

\subsection{Index Grids}

This is the trivial case: in the absence of any (geo) reference, direct positions are simply given by the array indices. For each such index axis, therefore, only the lower and upper bounds are required as stepping is 1 .

\subsection{Regular Referenced Grids}

The next case is a linear dependency between array indices and projected coordinates. This is the case, for example, with orthorectified imagery and many types of climate simulation output data. To model this, we introduce a regular axis which, in addition to the CRS axis definition mentioned earlier, contains origin (i.e., the lower boundary of the grid along this dimension), upper bound, and the constant stepping, commonly called resolution. In the grid shown in Fig. 3 top left, both axes are regular.

\subsection{Irregular Referenced Grids}

Next, we generalize from regular to irregular axes by abandoning the equidistance constraint; an example is shown in Fig. 3 bottom left. Consequently, for each coverage axis we need to provide the respective coordinate components of the direct positions, effectively extending the domain set from a single resolution value to a list of positions. To avoid singularities which might break some implementations we require this list to be strictly monotonous.

\subsection{Distorted Axis Nests}

In the previous case, coordinate components along the direct position vectors still are linearly independent. Sometimes, however, a grid is distorted in a way that each point needs to have its individual direct position stored explicitly. Consider Fig. 3

\footnotetext{
${ }^{1}$ The geometric dimension may be larger as the coverage may be embedded in some higher-dimensional space. One example is a 2D surface dataset, like an orthoimage, which is following the terrain and, hence, warped in 3-space.
}

bottom right. Obviously, in this case coordinate vector components cannot be associated with axes individually.

On a side note, this diagram as taken from GML 3.3 is misleading: drawing curves implies that a particular interpolation is possible. In fact, this is a separate issue which, in the coverage model proposed (cf. Conclusion section), is handled as part of the range type definition as only the range values "know" how they can be interpolated meaningfully.

We call such a situation, where a subset of the coverage's axes is intertwined, a nest of distorted axes. For a single distorted axis, this is equivalent to an irregularly spaced grid axis because the points remain on one and the same the axis. For a set of $1<m<d$ axes combined in a distorted subset (we call such a subset a "distorted grid"), this means that an $m$-dimensional array is needed to represent the coordinates of direct locations.

GML 3.3 supports this situation through its type ReferenceableGridByArray, albeit only for the special case where all grid axes, since they are treated equivalently, form a fully-specified distorted grid (i.e., $m=n$ ). A typical case where not all axes are engaged in a distorted grid is non-rectified satellite image timeseries: Lat and Long axes are distorted, whereas the time axis (while also "distorted") is independently specified; see Fig. 4 right for an example.

Differentiating such cases is important for an efficient storage: distorted grids occupy substantially more space than the irregular grids that do not have distorted axes. GML 3.3 shows this with the corresponding types where ReferenceableGridByVectors is much leaner than ReferenceableGridByArray.

Note that not all axes in a grid need to participate in a nest, and a grid may contain several disjoint nests (although this case is unlikely).

\subsection{Algorithmic Transformation Axes}

Up to now, cases have been considered where the mapping of the grid to an array can be described by some well-known formula. Any application can interpret (and, hence, transform) coordinates based on the coverage type and the ancillary information accompanying the grid. Sometimes, however, the transformation is given by a complex algorithm that depends on the type of sensing device and other physical parameters in a way that is too complex to represent in the coverage data structure; mathematical models for estimating geolocations from recorded camera data such as L0 swath imagery constitute a typical example. One method to describe such sensor models is established by SensorML 2.0 [11]. Such a sensor model involves two inputs: a sensor model description containing free variables plus a separate set of variable instantiations. As the sensor model defines the grid and its direct positions, this transformation effectively represents the coverage domain set.

\subsection{From Axes to Grids}

The axis types introduced allow modeling all quadrilateral ${ }^{2}$ grids - at the latest, distorted grids with their explicit representation and grids with some algorithmic transformation can describe any given grid. Axis types can be summarized as given by the UML diagram in Fig. 5 defining a GeneralGrid.

\footnotetext{
${ }^{2}$ In GIS terminology, a quadrilateral grid is an n-dimensional grid where every inner grid point has $2 d$ direct neighbours
} 
Following our approach, a concrete $d$-dimensional grid is built by lining up d axes, where each one can be of arbitrary type. This allows establishing the domain set of a coverage whereby the set of direct positions in the grid is determined by the axis semantics.

In the simplest case of a grid with index axes only, this is the product of the axis extents. For more complex grid types this computation gets more involved. For some grid $g$, let $n_{x}$ be the number of index axis elements, $n_{r}$ the number of regular axis elements, $n_{i}$ the number of irregular axis elements, $n_{d}$ the number of distorted axes, and $n_{t}$ the number of transformation axis items. Further, let the following positive integer numbers be defined for the number of direct position coordinates along axes or axis combinations:

- Index Axis: $p x_{a}:=$ g.a . upperBound - g.a.lowerBound + 1 for $a$ index axis in $g$;

- Regular Axis:

$p r_{a}:=\lfloor(g . a \cdot$ upperBound $-g \cdot a \cdot$ lowerBound +1$) /$ resolution $\rfloor$ (i.e., rounded down) for $a$ regular axis in $g$;

- Irregular Axis: $p i_{a}:=\operatorname{card}(g \cdot a$.directPositions $)$ for $a$ irregular axis in $g$;

- Distorted Axis: $p d_{d}:=\operatorname{card}(g . d$. directPositions $)$ for $d$ distorted axis in $g$;

- Transformation Axis: $p t_{m}:=\operatorname{card}(f(g))$ for $m \in g$.model where $f$ is a function on $g$ delivering all direct positions (such as a sensor model).

Then, the number $n_{p}$ of direct positions in $g$ is given by the product of all the above items:

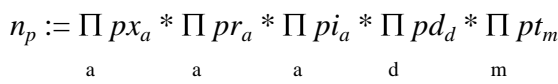

where a partial product is defined to be 1 if no such item exists.

Note that there is a hierarchy of increasing generality among these grid types. If we write " $<$ " for "is less general than", we obtain the following chain of grid types:
Grid

$<$ Rectified Grid

$<$ Irregular Grid

$<$ Distorted Grid

$<$ Transformation Grid

Obviously, this induces opportunities for data simplification: as the more specific, but memory-efficient axes can be modeled likewise through the more general, albeit high-volume alternatives a service might transform a "blown-up" representation losslessly into the most efficient one. An additional advantage is that having classes of increasing complexity allows implementations to decide individually how far they want to support the grid variants.

At this stage, we can come back to the examples shown in the introduction; these can be described schematically as in Fig. 6 . Let us consider the 4-D case in more detail. A coverage domain set can make use of this for describing a concrete grid. In a GML encoding, a sample 4-D domain set over a grid of Lat, Long, $h$, and date axes can be described as follows [3] (note the unit of measure labels, uomLabels, which indicate that Lat and Long are measured in degrees, height is measured in meters, and time in days). As described in Section 3, the CRS is construed from 3-D $\mathrm{x} / \mathrm{y} / \mathrm{z}$ CRS 4979, as defined in the EPSG list, and 1-D time CRS AnsiDate, as defined by OGC. For each of the four axes defined there is one regular or irregular axis element, resp.; for the regular axes, lower and upper bound together with resolution give a sufficient description whereas the irregular axes contain an explicit enumeration of the axis coordinates making up the grid. The last item in the grid is the description of the underlying array, which has axes $i, j, k$, and $l$ and a Cartesian CRS, Index4D.

\subsection{Processing}

The OGC Web Coverage Processing Service (WCPS) standard represents a declarative spatio-temporal geo raster language which supports queries on coverages, including the grids described in this paper; as space constraints do not allow to expand on this, so we refer to literature [5][7].

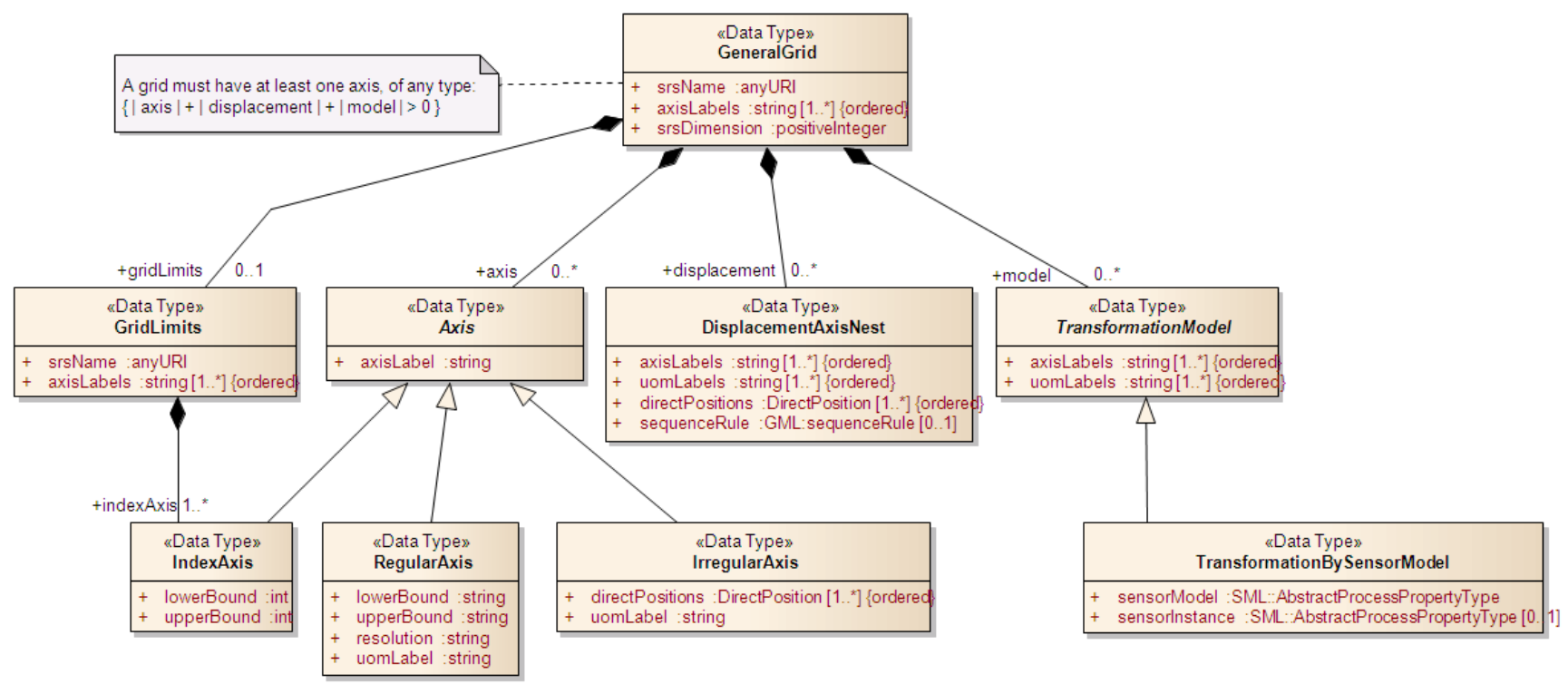

Fig. 5. UML diagram for the unified grid model [3] 


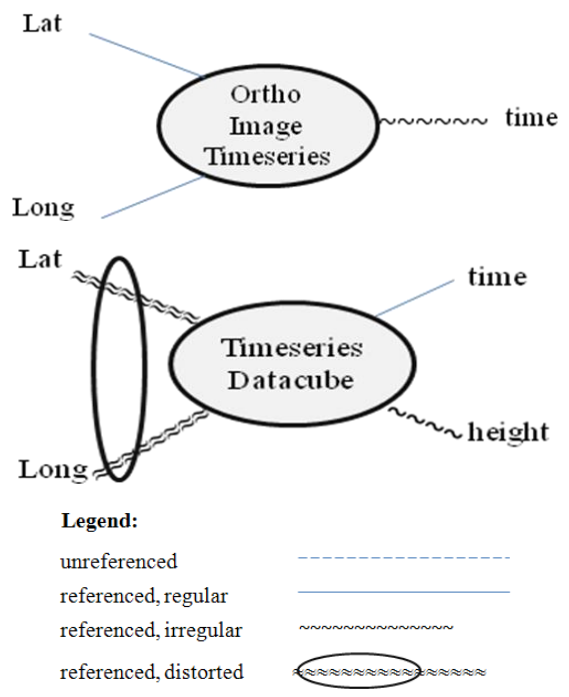

Fig. 6. Sample graphical 3-D and 4-D grid definitions

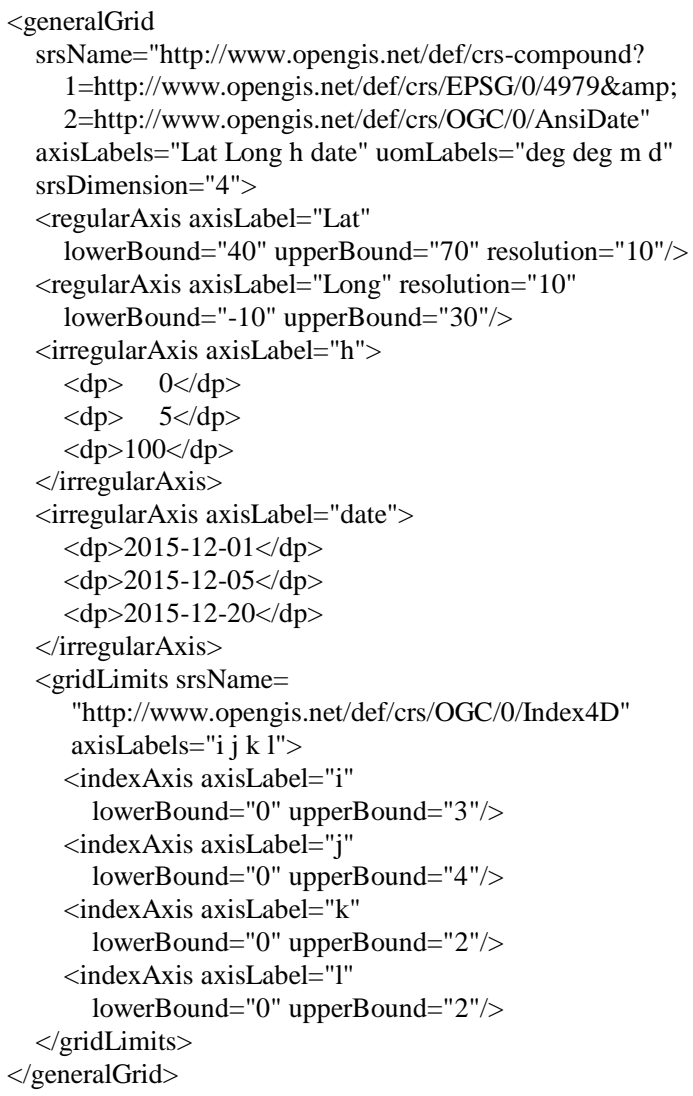

In Meteorology, different height axes are used frequently where a proxy value, such as pressure in hekto-Pascal, is used instead of measured height. Hence, the 3-D EPSG CRS cannot always be used as in this simplified example - another reason why concatenation of CRSs has been introduced by OGC.

\section{IMPLEMENTATION}

Regular, irregular, and distorted grids are already implemented on top of the rasdaman Array Database system as a geo semantics layer offering OGC WMS, WCS, and WCPS Web service interfaces. Pixel/voxel data are stored in the array database which only knows about index coordinates. Axis descriptions are stored relationally, with the exception of distorted nests which again are stored as arrays in rasdaman. This way, the data partitioning and parallelization capabilities of rasdaman (Fig. 7) can be used on the high-volume grids while the smaller-scale geo referencing information can be treated with relational queries.

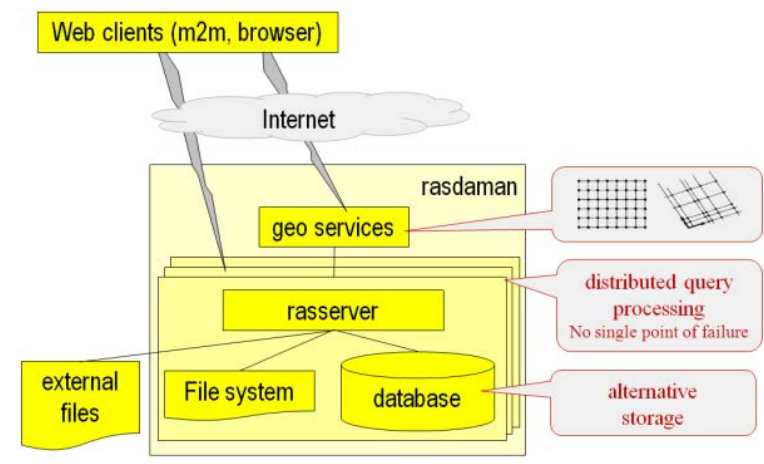

Fig. 7. rasdaman federated architecture

Rasdaman is used, e.g., in the EarthServer project where largescale data centers serve 3-D x/y/t satellite image timeseries and 4$\mathrm{D} x / \mathrm{y} / \mathrm{z} / \mathrm{t}$ weather datacubes as coverages based on the OGC WCS and WCPS standards [2]. Among others, the European Centre for Medium-Range Weather Forecast (ECMWF) is participating, its 90 PB archive being the largest meteorological archive worldwide. At the time of this writing, rasdaman database sizes exceed 100 TB [2], and queries have been parallelized automatically across more than 1,000 cloud nodes [17].

\section{CONCLUSION}

In this paper we have presented a unified model for grids with a quadrilateral topology, i.e., where every inner grid cell in a $d$ dimensional has exactly $2 d$ neighbors. Non-regular quadrilateral grids, while largely neglected in science, have a high practical relevance, ranging from raw swath data over elevation data to river and basin modeling.

The paper makes two original contributions to the field: first, modeling regular and irregular grids in a homogeneous manner which allows arbitrary combination of regular and irregular axes; second, a unified approach to handling both array and geodetic coordinates without restrictions on dimensionality, a sine-qua-non for services on such grids. Earlier attempts have either only considered regular grids, or have modeled irregular grids through informal textual descriptions. To the best of our knowledge, this is the first comprehensive tackling of grid modeling.

The approach to axis-centric grid modeling presented in this paper is under adoption vote by OGC as Coverage Implementation Schema (CIS) 1.1 [3]. ISO has commenced work on adopting this specification as 19123-2.

An open research issue is to transcend quadrilateral grids and find convenient mapping schemes for triangular and hexagonal grids, such as in simulation [32]. More generally, the question is how to represent, without undue additional conceptual burden, grids which have a fixed topology where all grid points share the same number of direct neighbors.

With regard to implementation, we plan to ruggedize the proof-ofconcept implementation of selected non-regular grid types into full support of the specification by the rasdaman engine. 


\section{ACKNOWLEDGEMENTS}

The authors gratefully acknowledge community feedback from the public OGC Request for Comment (RFC) phase. The first author additionally thanks the rasdaman team for its brilliant implementation work, and the EarthServer partners for valuable insights into large-scale geo datacube modeling.

\section{REFERENCES}

[1] ISO: 19123 Geographic information - Schema for coverage geometry and functions. ISO 19123:2004

[2] P. Baumann, P. Mazzetti, J. Ungar, R. Barbera, D. Barboni, A. Beccati, L. Bigagli, E. Boldrini, R. Bruno, A. Calanducci, P. Campalani, O. Clement, A. Dumitru, M. Grant, P. Herzig, G. Kakaletris, J. Laxton, P. Koltsida, K. Lipskoch, A.R. Mahdiraji, S. Mantovani, V. Merticariu, A. Messina, D. Misev, S. Natali, S. Nativi, J. Oosthoek, J. Passmore, M. Pappalardo, A.P. Rossi, F. Rundo, M. Sen, V. Sorbera, D. Sullivan, M. Torrisi, L. Trovato, M.G. Veratelli, S. Wagner: Big Data Analytics for Earth Sciences: the EarthServer Approach. Intl. Journal of Digital Earth 0(0)2015

[3] P. Baumann, E. Hirschorn: OGC Coverage Implementation Schema (CIS) version 1.1. OGC 09-146r3, 2016, http://external.opengeospatial.org/twiki public/pub/Coverag esDWG/CoveragesBigPicture/cis_1-1.zip; seen 2016-mar-20

[4] P. Baumann: A Database Array Algebra for Spatio-Temporal Data and Beyond. Proc. Next-Generation Inf. Sys. Tech. (NGITS), LNCS 1649, Springer 1999, pp. 76-93

[5] P. Baumann: The OGC Web Coverage Processing Service (WCPS) Standard. Geoinformatica, 14(4)2010, pp 447-479

[6] P. Baumann: OGC Web Coverage Service (WCS) - Core. OGC 09-110r4, https://portal.opengeospatial.org/files/09110r4; seen 2016-mar-20

[7] P. Baumann: OGC Web Coverage Processing Service (WCPS) Interface Language Standard. http://www.opengeospatial.org/standards/wcps, seen 201506-22

[8] P. Baumann: OGC GML 3.2.1 Application Schema - Coverages, version 1.0. OGC 09-146r2; https://portal.opengeospatial.org/files/?artifact_id=48553, seen on 2015-06-20

[9] P. Baumann: On the Management of Multidimensional Discrete Data. VLDB Journal 4(3)1994, Special Issue on Spatial Database Systems, pp. 401 - 444

[10] J.D. Blower, A.L. Gemmell, G.H. Griffiths, K. Haines, A. Santokhee, X. Yang: A web map service implementation for the visualization of multidimensional gridded environmental data. Env. Modelling \& Software, 47(0):218 - 224, 2013.

[11] M. Botts, A. Robin: OGC® SensorML: Model and XML Encoding Standard, version 2.0. OGC 12-000, 2012

[12] J.P. Boyd: A Fast Algorithm for Chebyshev, Fourier, and sinc Interpolation onto an Irregular Grid. Journal of Computational Physics, 103(2)1992, pp.243-257

[13] OGC: Compliance Interoperability \& Testing Evaluation SC. http://www.opengeospatial.org/projects/groups/citesc, seen 2016-mar-20

[14] OGC: CRS Name Type Specification, version 1.0. OGC 11135,2011
[15] J. de la Beaujardiere: OGC Web Map Server (WMS) Implementation Specification, version 1.3. http://www.opengeospatial.org/standards/wms; seen 2016mar-20

[16] DLR: DELIGHT Bathymetric Modelling of the Yellow River Delta Estuary. http://www.delight.eoc.dlr.de/en/node/329; seen 2016-mar-20

[17] A. Dumitru, V. Merticariu, P. Baumann: Exploring Cloud Opportunities from an Array Database Perspective. Proc. ACM SIGMOD Workshop on Data Analytics in the Cloud (DanaC'2014), June 22 - 27, 2014, Snowbird, USA

[18] M. Folk, G. Heber, Q. Koziol, E. Pourmal, D. Robinson: An Overview of the HDF5 Technology Suite and Its Applications. Proc. EDBT/ICDT 2011 Workshop on Array Databases, ACM 2011, pp. 36-47

[19] J. Hemphill: Geometry of Aerial Photography. http://www.geog.ucsb.edu/ jeff/115a/lectures/geometry_of_a erial_photographs_notes.html, seen 2016-mar-20

[20] E. Hirschorn, P. Baumann: OGC Testbed 11 Referenceable Grid Harmonization Engineering Report. OGC 15-065r1, 2015

[21] B. Howe, D. Maier: Algebraic Manipulation of Scientific Datasets. VLDB Journal, 14(4)2005, 397-41

[22] J. Mennis, R. Viger, C.D. Tomlin: Cubic Map Algebra Functions for Spatio-Temporal Analysis. Cartography and Geographic Information Science, 32(1)2005, pp.17-32

[23] D. Misev, P. Baumann: Extending the SQL Array Concept to Support Scientific Analytics. Proc. Intl. Conf. on Scientific and Statistical Database Management (SSDBM'2014), June 30 - July 2, 2014, Aalborg, Denmark, paper \#10

[24] N. n.: netCDF. http://www.unidata.ucar.edu/software/netcdf/; seen 2016-mar-20

[25] N. n.: SciDB. http://www.scidb.org, seen 2016-mar-20

[26] OGC: OpenGIS Abstract Specification Topic 6: Schema for coverage geometry and functions, version 7.0. OGC 07-011; http://portal.opengeospatial.org/files/?artifact_id=19820, seen on 2015-06-20

[27] V. Pekar, E. Gladilin, K. Rohr: An Adaptive Irregular Grid Approach for 3D Deformable Image Registration. Physics in Medicine and Biology 51.2 (2006): 361

[28] C. Portele: Geography Markup Language (GML) Encoding Standard, version 3.2.1. OGC 07-036, 2007

[29] C. Portele: OGC $^{\circledR}$ Geography Markup Language (GML) Extended schemas and encoding rules, version 3.3. OGC 10129r1, 2011

[30] WCS. project homepage at http://www.opengeospatial.org/ standards/wcs; seen on 2016-mar-20

[31] A. Settari, K. Aziz: Use of Irregular Grid in Reservoir Simulation. Society of Petroleum Engineers Journal, 12(02)1972, pp.103-114

[32] W.C. Thacker: A brief review of techniques for generating irregular computational grids. International Journal for $\mathrm{Nu}$ merical Methods in Engineering 15( 9)1980, pp. 1335-1341

[33] C.D. Tomlin: A Map Algebra. Harvard Graduate School of Design, 1990 